\title{
Arranging Icons on Small Displays: Do Hexagonal Layouts Improve Search Performance?
}

\author{
Tassilo Bouwman, Duncan P. Brumby \\ UCL Interaction Centre, University College London, London, UK
}

\begin{abstract}
Mobile devices with small displays can use hexagonal layouts of circular icons to use space more efficiently - we test whether this design choice improves people's search performance. An experiment is reported in which 24 participants performed known-item searches of menus that varied along four dimensions: (1) whether icons were arranged using a hexagonal or a grid layout, (2) how closely icons were placed next to each other, (3) the number of icons in the display that shared the same color as the target, and (4) whether icons stayed in the same location or moved between trials. Results show that search times were faster with hexagonal layouts but only when there were many same-color distractors and icons were packed very close to each other. This work contributes new empirical findings on how icon arrangements, in interaction with other critical visual design features, affect search performance.
\end{abstract}

\section{INTRODUCTION}

With the emergence of smartwatches and other wearable mobile devices, hexagonal layouts of circular icons have become increasingly popular. For example, the app launcher of the Apple Watch uses a hexagonal layout (see Figure 1). A hexagonal layout is a grid of circles that are arranged in a hexagonal pattern similar to a honeycomb. This layout represents a departure from using a grid layout with square icons, which is commonly used on desktop computers and smartphones. The benefit of the hexagonal layout is that circular icons can be packed closer together; it is the densest way of arranging circles in two dimensions (Williams, 1979). This makes hexagonal layouts ideal for devices with very small display screens.

Despite the adoption of hexagonal layouts for some devices with small displays, there has been very limited research to understand how people interact with these layouts. An in-depth observational study by Pizza, Brown, McMillan, and Lampinen (2016), which video-recorded users' everyday interactions with an Apple Watch, captured many instances in which users spent a long time searching the app launcher trying to locate a specific app icon. So, while hexagonal layouts can enable more icons to be displayed on a small screen, this layout design might hinder visual search because it creates a cluttered display (Everett \& Byrne, 2004; Lindberg $\&$ Nasanen, 2003). Might the use of a densely packed hexagonal layout be preventing users from quickly finding content on their smartwatch? Previous research has shown that using a densely packed display with small icons can negatively affect search time and accuracy (Schröder \& Ziefle, 2006). However, this study was conducted on a desktop computer using a grid layout of black and white icons, which severely limits the generalizability of these findings to understand how people search densely packed hexagonal layouts containing contemporary app icons that make extensive use of color.

This paper investigates whether hexagonal icon layouts improve or hinder visual search performance. To investigate this question, we conducted an online experiment using a custom-built smartphone app. The primary focus of the study

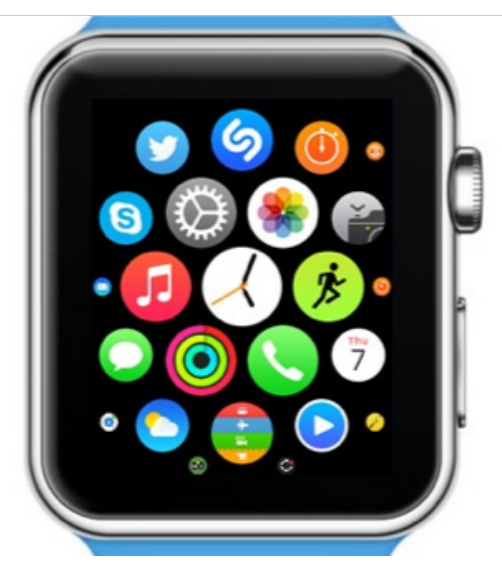

Figure 1: Hexagonal icon arrangement on the Apple Watch

was to investigate whether search performance was better with hexagonal icon layouts or traditional grid layouts for arranging circular icons on a display. We do not consider this factor in isolation, but instead how it interacts with several other key design features of menus. For example, the distance between icons (i.e., how closely they are packed next to each other), the number of icons in the menu that share the same color as the target that is being searched for (i.e., number of same-color distractors), and whether icons remained in a constant and stable location in the menu between searches (i.e., allowing location learning to occur). These features have been previously investigated for standard grid layouts of square icons (Lindberg \& Nasanen, 2003; Scarr, Cockburn, Gutwin, \& Bunt, 2012; Scarr, Cockburn, Gutwin, \& Malacria, 2013; Schröder \& Ziefle, 2006) and for linear word menus (Bailly, Oulasvirta, Brumby, \& Howes, 2014; Cockburn, Gutwin, \& Greenberg, 2007), but the impact of these features for small displays with hexagonal icon layouts is currently unknown. The results of this study will give an answer to this question and in doing so will provide empirical findings on how icon arrangements, in interaction with other critical design features, affect search performance. 


\section{METHOD}

\section{Participants}

Twenty-four participants (7 male) aged between 18 and $46(M=26, S D=6.97)$ were recruited through (https://www.callforparticipants.com) and social media. Age and gender were self-reported. Participants were entered into a raffle with Amazon vouchers worth $£ 20$ (GBP) as prizes. To ensure participants were independent a unique identifier was generated based on the participants iCloud account; an analysis of the data revealed no duplicate participants. The display size of each participant's device was automatically logged.

\section{Design}

The experiment used a $2 \times 2 \times 2 \times 2$ (layout $\times$ spacing $\times$ color $\times$ location learning) mixed design. Layout and color were manipulated as within-subjects factors. Participants searched icons arranged in a hexagonal layout and a grid layout. The order in which the layouts were searched was counterbalanced across conditions. The icon color factor was manipulated by using targets that shared the same color with either one or five distractor icons within the layout. Spacing and location learning were manipulated as between-subjects factors. The distance between icons was either zero for one half of the participants or at least 13.33 points apart for the other half. To allow location learning to occur, half of the participants searched menus in which icons stayed in the same position from one trial to the next. Performance in this stable condition was compared to that of participants who searched menu in which the position of each icon changed within the display from one trial to the next.

\section{Materials}

Participants searched icon menus. Each menu consisted of 24 icons that were arranged in a $6 \times 4$ layout. All icons had white unique foreground shapes and a solid background color (see Figure 2). Six icons were pink, six orange, and six blue. Two icons were green, two purple, and two turquoise. The distribution of colors was the same for all participants across all conditions to avoid confounding effects. To maintain the distribution of icon colors in the random condition the icons were shuffled in a controlled way. With every new trial the three top and bottom rows were interchanged, and the icons were shuffled within each row.

For each participants the foreground shapes were randomly assigned to a color to minimize any potential confounding effects of shape on search performance. The position of each icon was the same for all participants in the stable condition. In the random condition the position of each icon changed with every new trial. Stimuli were presented on a white background with a status bar that showed the number of trials left to complete and a cancel button that allowed participants to stop the experiment at any point.

The ratio of icon size and distance was picked based on the iPhone's home screen icons for ecological validity. The
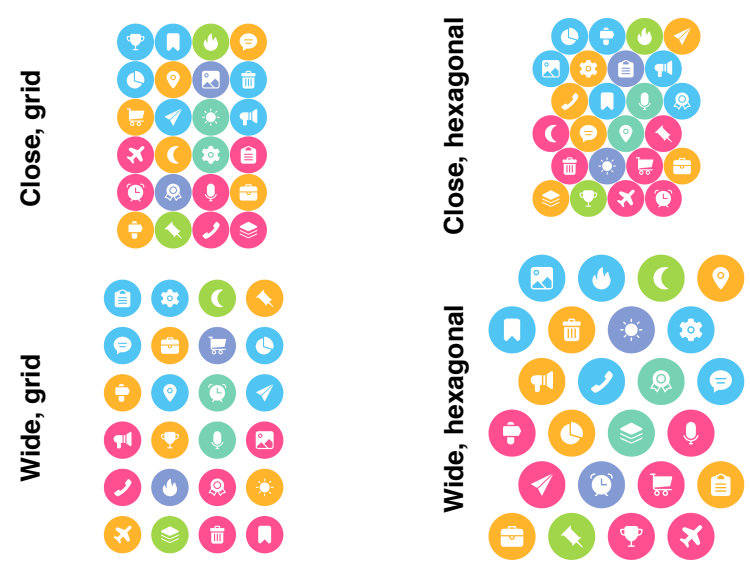

smallest iPhone's icon size was 60 points with a horizontal distance between each icon of 16 points (15:4 ratio). An icon size of 55 was chosen to fit the hexagonal layout on the smallest iPhone screen. Thus, a distance of 13.33 points was used for the wide distance condition to maintain the 15:4 ratio. The distance for the close distance condition was one point. The icon size and distances of each condition were the same for all iPhone display sizes. For all iPads the icon size was increased by quarter of the iPhone's icon size because iPads are usually viewed from a greater distance. Thus, the perceived icon size remained similar to iPhones. The icon size to distance ratio was kept the same for all devices.

A mobile application was developed to conduct the experiment. The application consisted of three sections: dashboard, activities and profile. The dashboard showed the average search time for each completed activity. The purpose of the dashboard was to motivate participants completing the tasks by allowing them to reflect on their performance. In the activities section, three search tasks, a survey task, and a task to sign up for a reward were shown in a list. A task could be launched by tapping the entry in a list. Only one task was available at a time. The purpose of presenting all tasks was to give participants an overview of what was expected from them. In the profile section the participant's ID, the assigned group, and the name of the study were displayed. In addition, participants could export their consent form and result files and withdraw from the study if they wanted to. The purpose of this section was to provide participants control over the data they share. Due to time constraints the app was made for iOS devices only.

\section{Procedure}

Participants downloaded the app on the Apple App Store, launched the app and completed a consent form. An introduction screen informed participant about the task: participants had to locate a given target icon in a menu as quickly as possible. At the start of each trial participants were shown the icon they were looking for. After seeing the icon participants started the trial by clicking the 'Next' button. This made the target icon and the button disappear by sliding out to 
the left while the icon menu appeared by sliding in from the right. Participants selected an icon by tapping it using a finger. If an incorrect selection was made, the icon menu disappeared, and the icon of the same target reappeared so participants could repeat the trial. If participants selected the correct icon the trial ended, and they proceeded to the next trial.

Participants completed 63 trials in total. The first three trials were practice trials that were included to familiarize the participant to the selection task and how it operates. The remaining 60 trials were divided into two blocks. One block of 30 trials contained hexagonal layouts and one block of 30 trials contained grid layouts. The sequence in which these blocks appeared was counter-balanced between participants. Half of the participants performed all hexagonal trials before the grid trials, and vice versa. An introductory screen of the new layout appeared before the first trial of the second block started. Trials in which the participants did not select the correct target on the first attempt were recorded as errors.

Search time was recorded by the device by calculating the time difference between the appearance of the icon menu and the selection of the icon. The result was saved as one entity together with the participant's anonymous identifier and condition information. In addition, target description position, display size, and hours since the completion of the last trial were saved. At the end of the experiment all entities were written into a comma-separated values (CSV) file, encrypted and uploaded to a secure server which could only be accessed by the researchers.

\section{RESULTS}

A mixed ANOVA was used, with a significance level of .05 for judging the significance of effects. We excluded trials from the analysis in which the participant did not correctly select the target on the first attempt. Accuracy was generally very high - incorrect icons were selected on only $3.4 \%$ of trials (51 from a total of 1,512). A further 8 trials were excluded because of exceedingly slow response times (greater than 5 seconds). These outliers were three times the interquartile range above the third quartile.

The primary measure of interest was search time (i.e., how long it took participants to correctly locate and select the target icon). As expected, participants were significantly faster at locating targets when icons stayed in the same position in the menu across repeated trials $(M=1.36 \mathrm{~s}, S D=0.29 \mathrm{~s})$ compared to when the location of icons on the screen changed from one trial to the next $(M=1.56 \mathrm{~s}, S D=0.22 \mathrm{~s}), F(1,20)=$ $5.47, p<.05, \eta_{\mathrm{p}}^{2}=.16$. Participants were also significantly faster at locating targets when there was only one same-color distractor in the menu $(M=1.38 \mathrm{~s}, S D=0.21 \mathrm{~s})$ compared to when there were five same-color distractors $(M=1.54 \mathrm{~s}, S D=$ $0.31 \mathrm{~s}), F(1,20)=12.51, p<.05, \eta_{\mathrm{p}}{ }^{2}=.002$. There was no significant main effect of search time on either menu layout, $F(1,20)=3.09, p=.09, \eta_{\mathrm{p}}{ }^{2}=.01$, or icon distance, $F<1$. As we show below, the effect of menu layout was dependent on the number of same-color distractors and icon distance.

Figure 3 shows mean search time data for menu layout and icon distance, separated by whether menus had one samecolor distractor (left panel) or five same-color distractors

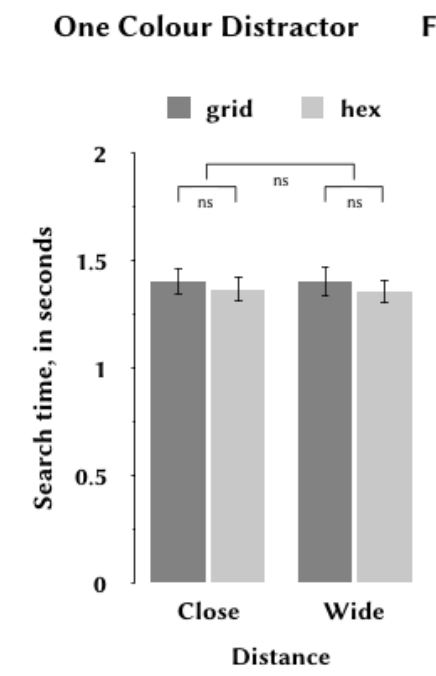

Five Colour Distractors

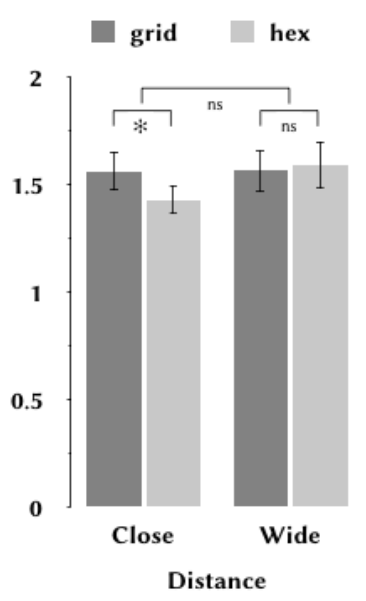

Figure 3: Mean search time with one color distractor (left) and five color distractors (right). Error bars depict standard errors.

$$
* p<.05 \text {, and n.s. is not significant. }
$$

(right panel). It can be seen in the left panel of Figure 3 that when menus contained only one same-color distractor search times were similar regardless of the menu layout and icon distance. Statistical analysis supports this observation, finding no interaction between distance and layout on search time, $F<1$.

The right panel of Figure 3 shows that when menus contained five same-color distractors the layout of the menu and distance between icons impacted search performance. There was a significant two-way interaction between distance and layout on search time, $F(1,20)=4.71, p=.04, \eta_{\mathrm{p}}{ }^{2}=.02$. Follow-up tests of this interaction revealed that when icons were packed close together, participants were significantly faster at locating targets arranged in a hexagonal layout $(M=$ $1.43 \mathrm{~s}, S D=0.23 \mathrm{~s})$ than a grid layout $(M=1.56 \mathrm{~s}, S D=0.30 \mathrm{~s})$, $F(1,11)=9.42, p=.01, \eta_{\mathrm{p}}{ }^{2}=0.06$. However, there was no significant effect of menu layout when items were spaced further apart in the wide condition, $\mathrm{F}<1$.

Finally, because participants used their own devices to take part in the experiment, we consider whether differences in the screen size affected the results. Nine participants used a 4 " display, seven a 5.8" display, five a 4.8" display, two a 9.7" display and one a 5.5" display. A mixed-design ANOVA with display size as a random effect was applied to the data. It was found that there was no significant effect of display size on search performance. Because of this display size was therefore excluded from the above analysis.

\section{DISCUSSION}

The findings of this study can help designers decide how to arrange icons on devices with small displays. There are three empirically-grounded recommendations that follow from this work: (1) devices should arrange icons using a hexagonal layout but only when there are many same-color distractors and icons were packed very close to each other, 
(2) dynamically moving icons within a display should be avoided as it hinders search, and (3) to help users find icons quicker, designers should expand the range of colors used. We discuss each of these recommendations in turn.

First, the results of this study show that participants were faster at locating targets when searching hexagonal layouts but only when there were many same-color distractors and icons were packed very close to each other. This finding would suggest that the current trend for using hexagonal displays on densely packed small displays, such as smartwatches and other wearable devices, helps users quickly identify the app icon that they are looking for.

Second, participants were faster at locating targets when icons stayed in the same position compared to when the location of icons changed from one trial to the next. This finding replicates and extends previous research

demonstrating a similar location learning effect in word-based command menus used in desktop computer (Bailly et al., 2014; Cockburn, Gutwin, \& Greenberg, 2007; Ehret, 2002; Treisman, 1982). The current study demonstrates that this location learning effect is robust and also occurs when people search for app icons on mobile devices. This finding suggests that designers should avoid dynamically moving icons within a display as it hinders visual search.

Third, participants were faster at locating targets when there was only one same-color distractor in the menu compared to when there were five same-color distractors. This finding is consistent with previous research demonstrating that colour is an important cue for guiding visual search (Gordon \& Abramov, 1977; Kieras \& Hornof, 2014; Yi, Yu, Shi, \& Shi, 2017). This study shows that color can lose its value as a cue for users to distinguish app icons on mobile devices if many icons use the same color. To help users finding app icons quickly designers should employ a variety of background colors.

\section{Limitations and Future Work}

A limitation of the study is that it was conducted on a smartphone rather than a smartwatch. This was done for three reasons. First, studies on text entry methods showed no significant difference between using a smartphone and a smartwatch (Pizza et al., 2016; Visuri, Sarsenbayeva, van Berkel, Goncalves, Rawassizadeh, Kostakos, \& Ferreira, 2017). Second, smartwatches are less common than smartphones, which makes it hard to find participants. Third, it is harder to deploy an experiment on smartwatches. Frameworks like ResearchKit, that enable easy implementation of visual search experiments, are not available for smartwatches. A potential workaround is to use a toolkit which simulates a smartwatch using smartphones (Houben \& Marquardt, 2015). In this approach a smartphone was placed on the users' arm and paper cut-outs were employed to simulate a watch display. However, such an approach is cumbersome to use and cannot be deployed remotely, which was one of our key requirements to reach a broad audience with our experiment. Future on-site lab experiments may use this toolkit to validate the results of the current student on watch displays.

\section{CONCLUSION}

The current study was designed to investigate search time of hexagonally arranged icons using a smartphone app. Results show that search time in hexagonal icon layouts depends on how close items were to each other. When items were packed close together, a hexagonal icon layout was searched faster than a grid layout. However, this layout effect was not detected when icons were spaced further apart from each other. These results were based on search data collected using a custom smartphone app that participants obtained from the Apple App Store. To help users finding app icons quickly designers should employ hexagonal icon layouts, use a variety of background colors, and keep the position of icons stable.

\section{REFERENCES}

Bailly, G., Oulasvirta, A., Brumby, D. P., \& Howes, A. (2014). Model of visual search and selection time in linear menus. In Proceedings of the 32Nd Annual ACM Conference on Human Factors in Computing Systems (CHI '14). ACM, New York, NY, USA, 3865-3874. DOI: $\underline{\text { ttp://dx.doi.org/10.1145/2556288.2557093 }}$

Cockburn, A., Gutwin, C., \& Greenberg, S. (2007). A predictive model of menu performance. In Proceedings of the SIGCHI Conference on Human Factors in Computing Systems (CHI '07). ACM, New York, NY, USA, 627-636. DOI: http://dx.doi.org/10.1145/1240624.1240723

Ehret, B. D. (2002). Learning where to look: location learning in graphical user interfaces. In Proceedings of the SIGCHI Conference on Human Factors in Computing Systems (CHI '02). ACM, New York, NY, USA, 211218. DOI: http://dx.doi.org/10.1145/503376.503414

Everett, S. P. \& Byrne, M. D. (2004). Unintended effects: varying icon spacing changes users' visual search strategy. In Proceedings of the SIGCHI Conference on Human Factors in Computing Systems (CHI '04). ACM, New York, NY, USA, 695-702. DOI: http://dx.doi.org/10.1145/985692.985780

Gordon, J. \& Abramov, I. (1977). Color vision in the peripheral retina. II. Hue and saturation*. Journal of the Optical Society of America 67(2), 202-207. DOI: http://dx.doi.org/10.1364/JOSA.67.000202

Houben, S. \& Marquardt, N. (2015). WatchConnect: a toolkit for prototyping smartwatch-centric cross-device applications. In Proceedings of the 33rd Annual ACM Conference on Human Factors in Computing Systems (CHI '15). ACM, New York, NY, USA, 1247-1256. DOI: http://dx.doi.org/10.1145/2702123.2702215

Kieras, D. E. \& Hornof, A. J. (2014). Towards accurate and practical predictive models of active-vision-based visual search. In Proceedings of the 32Nd Annual ACM Conference on Human Factors in Computing Systems (CHI '14). ACM, New York, NY, USA, 3875-3884. DOI: http://dx.doi.org/10.1145/2556288.2557324

Lindberg, T. \& Nasanen, R. (2003). The effect of icon spacing and size on the speed of icon processing in the human 
visual system. Displays 24(3), 111-120. DOI:

http://dx.doi.org/10.1016/S0141-9382(03)00035-0

Pizza, S., Brown, B., McMillan, D., \& Lampinen, A. (2016).

Smartwatch in vivo. In Proceedings of the 2016 CHI

Conference on Human Factors in Computing Systems

(CHI '16). ACM, New York, NY, USA, 5456-5469.

DOI: http://dx.doi.org/10.1145/2858036.2858522

Scarr, J., Cockburn, A., Gutwin, C., \& Bunt, A. (2012).

Improving command selection with CommandMaps. In Proceedings of the SIGCHI Conference on Human Factors in Computing Systems (CHI '12). ACM, New York, NY, USA, 257-266. DOI:

http://dx.doi.org/10.1145/2207676.2207713

Scarr, J., Cockburn, A., Gutwin, C., \& Malacria, S. (2013). Testing the robustness and performance of spatially consistent interfaces. In Proceedings of the SIGCHI Conference on Human Factors in Computing Systems (CHI '13). ACM, New York, NY, USA, 3139-3148. DOI: http://dx.doi.org/10.1145/2470654.2466430

Schröder, S. Ziefle, M. (2006). Icon design on small screens: effects of miniaturization on speed and accuracy in visual search. In Proceedings of the Human Factors and Ergonomics Society Annual Meeting, 50(5), 656-660. DOI: http://dx.doi.org/10.1177/154193120605000508

Treisman, A. (1982). Perceptual grouping and attention in visual search for features and for objects. Journal of experimental psychology. Human perception and performance 8 (2), 194-214. DOI: http://dx.doi.org/10.1037//0096-1523.8.2.194

Visuri, A., Sarsenbayeva, Z., van Berkel, N., Goncalves, J., Rawassizadeh, R., Kostakos, V., \& Ferreira, D. (2017). Quantifying sources and types of smartwatch usage sessions. In Proceedings of the 2017 CHI Conference on Human Factors in Computing Systems (CHI '17). ACM, New York, NY, USA, 3569-3581. DOI: http://dx.doi.org/10.1145/3025453.3025817

Williams, R. (1979). The Geometrical Foundation of Natural Structure: A Source Book of Design. Dover, New York, NY, USA.

Yi, X., Yu, C., Shi, W., \& Shi. Y. (2017). Is it too small?: investigating the performances and preferences of users when typing on tiny QWERTY keyboards. International Journal of Human-Computer Studies, 106, 44-62. DOI: http://dx.doi.org/10.1016/j.ijhcs.2017.05.001 